\title{
IMMITTANCE SPECTROSCOPY OF SMART COMPONENTS AND NOVEL DEVICES
}

\author{
MOHAMMAD A. ALIM \\ Hubbell Incorporated, The Ohio Brass Company, 8711 Wadsworth Road, Wadsworth, Ohio 44281 \\ SANJIDA KHANAM AND MARTIN A. SEITZ \\ College of Engineering, Marquette University, Milwaukee, Wisconsin 53233 \\ (Received December 6, 1993; in final form January 25, 1994)
}

\begin{abstract}
AC small-signal immittance spectroscopy is employed as a viable tool to demonstrate electrical characterization, performance improvement, and quality assurance issues of smart materials-based components and novel devices. The variation in the ac response, complemented via dc measurements within a range of tolerating temperature, delineates competing phenomena occurring in the microstructures of these engineering material systems. The results are presented in a generic manner with possible explanations on the mechanisms for two selected Debye-like (nearly ideal) and non-Debye (non-ideal) low-capacitance resistors. This spectroscopic approach allows systematic development of a representative equivalent circuit, considered to be the characteristic of the devices and components, for specific applications.
\end{abstract}

\section{INTRODUCTION}

A reliable characterizing tool for the electrical components and devices based on smart engineering material systems is necessary to establish a set of desired parameters for application purposes. These material systems, in general, refer to heterogeneous composites, hybrids, thick-films, surface mount, and grain-boundary controlled devices, etc. The rationale for their characterization is that a better understanding of the nature of the smart materials serves as a vehicle by which processing can be related to the properties required for a particular application. Various physical, mechanical, chemical, microstructural, and electrical means of characterization are able to yield complementary information concerning the nature of materials. In terms of heterogeneous materials used in electrical components, the latter three are of significant interest. A number of factors are found to influence the electrical behavior of materials. These include the mechanisms of conduction and polarization, conduction current paths, and regions of significance within these paths. ${ }^{1}$ In this regard, the non-destructive ac electrical measurement techniques are of particular utility in that the factors being probed in specimens under test are usually the same as those operative in the components constructed of these heterogeneous materials.

It is evident that immittance (impedance or admittance) spectroscopy (IS) has proven to be a useful means of characterizing the electrical nature of a number of complex heterogeneous (or multi-component) systems. ${ }^{1-5}$ This tool unravels un- 
derlying competing phenomena via lumped parameter/complex plane analysis (LP/CPA $)^{1-8}$ and Bode plane analysis (BPA). ${ }^{9}$ These analytical techniques reveal structural non-uniformity, variation in conduction paths, role of defect states, degradation, stability, time-dependent processes, etc., in the heterogeneous materialbased components and devices. The LP/CPA technique also elucidates, for example, inter-particle electrical barriers, origin of the resistance or capacitance, and associated temperature coefficient of resistance (TCR) for the low-capacitance resistors (LCRs). The lumped parameter equivalent circuit modelling of an unknown device/material system (device under test-DUT) can be of assistance in the development of an electrical component.

An equivalent circuit consisting of the elements (resistor, capacitor, etc.) representing underlying mechanisms in the possible multi-component systems may be described for the observed electrical behavior as a function of temperature. Furthermore, LP/CPA and BPA techniques provide solutions to several problems related to the properties of material systems and the design of either a resistor or a capacitor. This powerful technique is a reliable resource for investigating complex material systems and their designs related to performance improvement and quality assurance issues. Finally, this approach allows the development of an equivalent circuit representation of the total ac response, which incorporates underlying mechanisms in a systematic manner under a given set of experimental conditions. ${ }^{1,2,6-8}$

The objective of this work is to focus both characterization approaches in the development stages, and reproducibility of the associated parameters at the manufacturing environment of the microelectronic resistive systems utilizing the IS approach. The material systems constituting the components considered for the demonstration is not known and not essential. The manufacturer can decide the constituents of the components for specific applications. As an example of case studies, an approach to the analysis of the ac small-signal electrical data as a function of frequency is provided for two types of LCRs. These LCRs belong to the Debyelike (nearly ideal) and non-Debye (non-ideal) classes due to the nature of the conducting paths operative between the terminal electrodes.

\section{LUMPED REPRESENTATION OF SIMULTANEOUSLY COMPETING PHENOMENA}

It is essential to possess a general concept on the nature of the electrically conductive paths and associated operative phenomena for a DUT before performing complete characterization processes. A DUT is likely to be more heterogeneous and complex than a single-phase crystalline system. In general, a single-crystalline material is less complex system in determining the resulting performance characteristics. The heterogeneous systems, in many cases, are polycrystalline or amorphous in nature consisting of multiple phases in the microstructures. The resulting arrangement of the grains, grain boundaries, and other phases constitute an integrated seriesparallel lacy network of conducting paths involving " $m$ " junctions in parallel and " $n$ " junctions in series between the electrodes. ${ }^{2}$ In the microstructural conduction 
modelling the easiest (i.e., shortest) routes constitute the electrical paths. Thus, the grains and identical relevant phases must lump in series with the grain boundaries since the inter-connecting junctions parallel (or nearly parallel/horizontal) to the electrode surface do not constitute the shortest electrical path. This series equivalent electrically conducting path, assumed to be perpendicular (or nearly perpendicular/vertical) to the electrode surface, must be operative between the two terminal electrodes without the influence of the cross-linked series-parallel lacy network in the microstructure. Therefore, the possible interference from the crosslinked lacy network is assumed to be extremely negligible, and may have either no or very weak effect on the parallel conducting paths simultaneously operative between the two terminal electrodes.

It is convenient to represent a DUT in the form of a lumped parameter equivalent circuit as is done with other electrical components such as: $p-n$ junctions, metalsemiconductor diodes, transistors, MOS/MIS devices, etc. This is achieved by following a set of rules ${ }^{1}$ that can be summarized as follows:

1. each significant conduction path must be represented by a parallel equivalent circuit path (Figure 1a);

2. each significant charge carrying species must be represented by a parallel equivalent path (Figure $1 b$ );

3. each region of significance within a path must be represented by a lumped parameter circuit with respect to the type of charge carrying species (Figure $1 c)$

4. each mechanism of polarization and relevant phenomena must be represented by either a series or parallel combination of resistors, capacitors, and/or inductors;

5. the components representing various conducting species and polarization processes each have their own composition, microstructural, temperature, voltage, pressure, environment, and frequency dependence;

6. the electrical paths of significance are those yielding the least impediment to current flow;

7. the regions of significance within these electrical paths are those presenting the largest impediment to current flow; and

8. complex plane plotting techniques can be used to elucidate an appropriate equivalent circuit representation from measured electrical quantities when used in conjunction with information obtained from other methods of study.

Additional contribution from the electrode material and its possible interaction with the DUT's surface must be in series with the combined series behavior of the grains, grain boundaries, and other phases. The physical location of each of these phases in the microstructure between the measurement terminals represents the net series effect. Figure 1 illustrates the lumped representation of the foregoing discussion, incorporating simultaneously operative competing phenomena, in an electrically active heterogeneous system. 

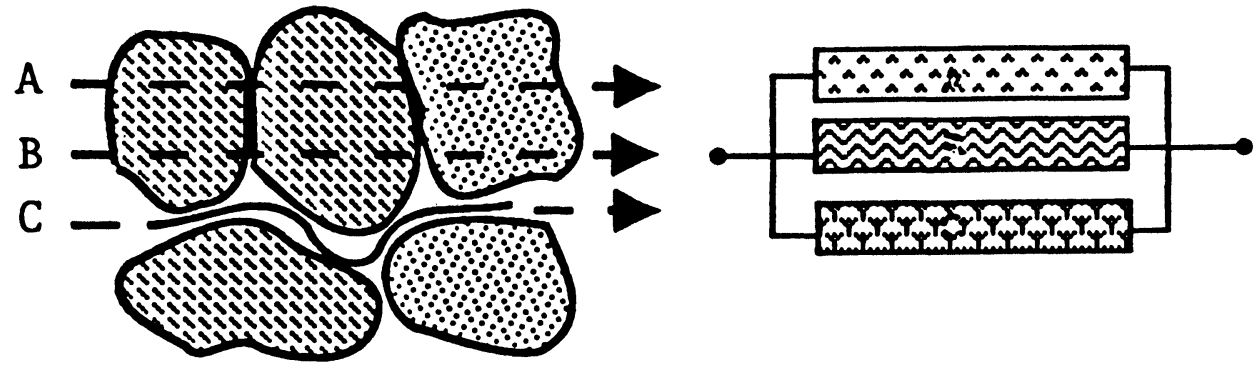

a. Each significant conduction path must be represented by a parallel equivalent circuit path.

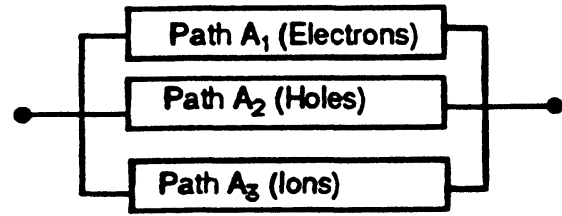

b. Each significant charge carrying species must be represented by a parallel equivalent path.

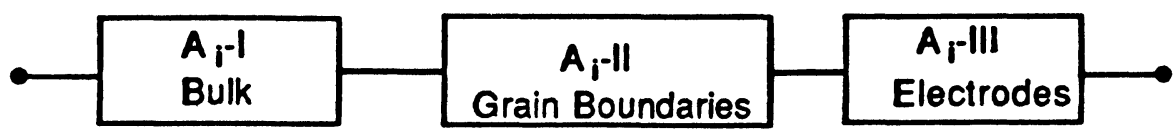

c. Each region of significance within a path must be represented by a lumped parameter circuit with $\mathrm{i}=1,2$ and 3 (Figure b) for the type of charge carriers.

FIGURE 1 Illustration of the lumped representation incorporating simultaneously operative competing phenomena in an electrically active heterogeneous system.

\section{LUMPED PARAMETER/COMPLEX PLANE AND BODE PLOTS}

The LP/CPA and BPA techniques employ the acquisition of the two-terminal ac small-signal electrical data (Figure 2) of a DUT in any one of the following three forms as a function of applied frequency $(f)$ :

1. impedance or series (a resistor, $\mathrm{R}_{\mathrm{s}}$, in series with a capacitor, $\mathrm{C}_{\mathrm{s}}$ );

2. admittance or parallel (a resistor, $R_{p}$ or a conductor, $G_{p}$, in parallel with a capacitor, $\left.\mathrm{C}_{\mathrm{p}}\right)$; and

3. phasor (magnitude of immittance and the corresponding loss tangent, tan $\delta$, or phage angle, $\delta$ ).

The measurement will be useful in any one form. The choice is dependent on the material characteristic and resolution capability of the measuring components provided by the instruments at a particular frequency range. To reduce the stray and lead effects it is better to keep shorter contact leads. The small-signal amplitude must be adjusted to achieve satisfactory data. The reproducibility of the data 


\section{A. Preferred Measured Forms}

(a) Series:

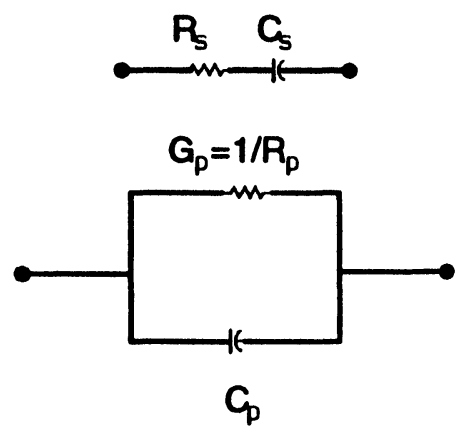

(c) Phasor: $\left|\mathrm{Z}^{*}\right|$ or $\left|\mathrm{Y}^{*}\right|$ and loss tangent $\left(\tan \delta=\mathrm{G}_{\mathrm{p}} / \omega \mathrm{C}_{\mathrm{p}}=\omega \mathrm{R}_{\mathrm{s}} \mathrm{C}_{\mathrm{s}}\right)$

B. Four Complex Plane Equations $\left(\omega=2 \pi f, f=\right.$ measured frequency, $\left.j=(-1)^{1 / 2}\right)$

1. Impedance: $Z^{*}=R_{s}-j\left(1 / \omega C_{s}\right)$

2. Admittance: $\mathrm{Y}^{*}=\mathrm{G}_{\mathrm{p}}+\mathrm{j} \omega \mathrm{C}_{\mathrm{p}}$

3. Capacitance: $\mathrm{C}^{*}=\mathrm{C}_{\mathrm{p}}-\mathrm{j}\left(\mathrm{G}_{\mathrm{p}} / \omega\right)$

4. Modulus: $\quad M^{*}=1 / C_{s}+j \omega R_{s}$

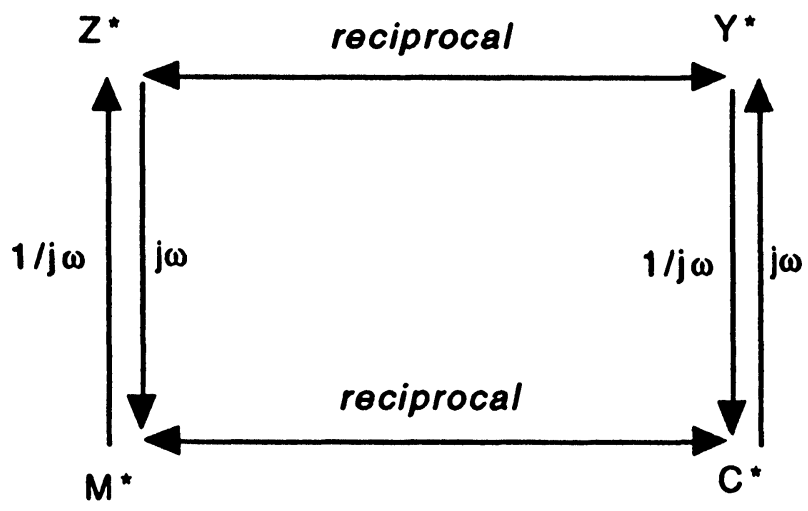

Fundamental equations in four complex planes representing the measured form of data and its transformation related to electrical characteristics consisting of $Z^{*}, \mathrm{Y}^{*}, \mathrm{C}^{*}$ and $\mathrm{M}^{*}$. Arrows imply multiplying factors for conversion between the complex planes.

FIGURE 2 Choice of measured forms of the acquisition of the ac small-signal electrical data and inter-convertible relationships within the four complex planes.

depends on sufficient modulation of the quasi-equilibrated Fermi-level for the testsignal amplitude in the DUT. Often the ultra-low signal amplitude may provide a misleading set of data containing noise with poor reproducibility. The polarization effect at low and ultra-low frequencies also yields noise and scattering of the data. In this case, enough equilibration time is necessary to acquire the data. The utilization of the fast Fourier transform (FFT) is a common practice to avert such problems. ${ }^{7.8}$ 
The measured impedance $\left(\mathrm{Z}^{*}\right)$ and admittance $\left(\mathrm{Y}^{*}\right)$ as a function of the ac smallsignal frequency consisting of series and parallel parameters operative in-phase (constituting real part) and out-of-phase (constituting imaginary part), respectively, are expressed by:

$\mathrm{Z}^{*}=\mathrm{R}_{\mathrm{s}}-j \frac{1}{\omega \mathrm{C}_{\mathrm{s}}}$,

and

$\mathrm{Y}^{*}=\mathrm{G}_{\mathrm{p}}+j \omega \mathrm{C}_{\mathrm{p}}$

where $j=\sqrt{-1}$, angular frequency $\omega=2 \pi f$, and $\mathrm{R}_{\mathrm{s}}, \mathrm{C}_{\mathrm{s}}, \mathrm{G}_{\mathrm{p}}$, and $\mathrm{Cp}$ are measured series resistance, series capacitance, parallel conductance, and parallel capacitance, respectively. The phasor data also translate to either form of the above mentioned two equations. As an example, the phasor form may be expressed for $\mathrm{Z}^{*}$ as:

$\mathrm{Z}^{*}=\left|\mathrm{Z}^{*}\right| \sin \delta+j\left|\mathrm{Z}^{*}\right| \cos \delta=\left|\mathrm{Z}^{*}\right| e^{(j \delta)}=\frac{\mathrm{V}}{\mathrm{I}}<\delta$,

where $\left|Z^{*}\right|$ is the absolute modulus (magnitude) of $\mathrm{Z}^{*}, \delta$ is the phase angle between the voltage, $\mathrm{V}$, across, and current, I, through the sample. After the acquisition of the ac electrical data, analysis comes next and they may be performed via:

1. lumped parameter/complex plane plot;

2. Bode plot; and

3. spectroscopic plot.

To perform the LP/CPA and/or BPA, it is convenient to use the data either in the form of impedance or admittance. An equivalent circuit representation is facilitated by displaying these data in the form of complex plane plots. A trial analysis in four complex planes is necessary for the as-measured immitance data. These plots, when combined with other known features of the system under study, allow an appropriate equivalent circuit representation of the system. The equivalent circuit model can be used to delineate the contribution of various regions of the microstructure on the DUT's electrical behavior and influence of process variables on these regions. The complex planes are inter-convertible (Figure 2), and incorporating geometrical factors they are summarized below with their equivalent synonyms:

1. impedance $\left(\mathrm{Z}^{*}\right)$ or resistivity $\left(\rho^{*}\right)$;

2. admittance $\left(\mathrm{Y}^{*}\right)$ or conductivity $\left(\sigma^{*}\right)$;

3. capacitance $\left(\mathrm{C}^{*}\right)$ or permittivity $\left(\epsilon^{*}\right)$; and

4. modulus $\left(\mathrm{M}^{*}\right)$ or electric-modulus $\left(m^{*}\right)$.

Each of these complex planes is suited to the representation of materials with certain particular characteristics, and may be obtained from the acquisition form 
of the data shown in Figure 2. The direction of vertical arrows indicate the multiplication factor $j \omega$ (or $1 / j \omega$ ) to be used for the conversion purposes of the associated pair of parameters. The horizontal arrows indicate reciprocal relationship between the relevant pair of parameters. The inter-convertible relationship among these complex planes exists in the following way:

$\mathrm{Z}^{*}=\frac{1}{\mathrm{Y}^{*}}, \quad \mathrm{C}^{*}=\frac{\mathrm{Y}^{*}}{j \omega}, \quad$ and, $\mathrm{M}^{*}=\frac{1}{\mathrm{C}^{*}}=j \omega \mathrm{Z}^{*}$.

The sample plots with associated simplified equivalent circuit representation for each of the four complex planes are depicted in Figure 2. A single semicircular relaxation in any of these complex planes will lead to a meaningful interpretation. It should be noted that ordinate ( $y$-axis) and abcissa ( $x$-axis) must have the same scale. Each complex plane plot represents a simple equivalent circuit model resulting from the semicircular relaxation and the intercept on the $x$-axis. An ideal

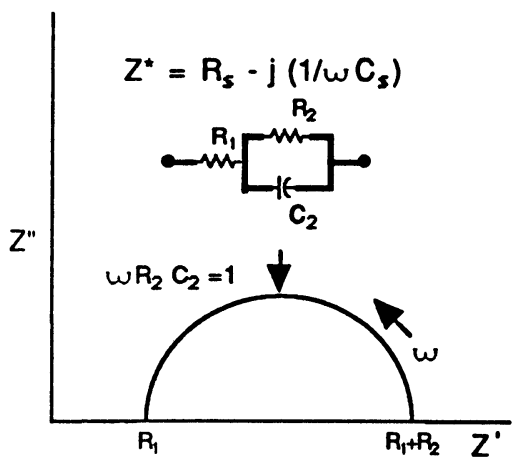

Impedance Plot

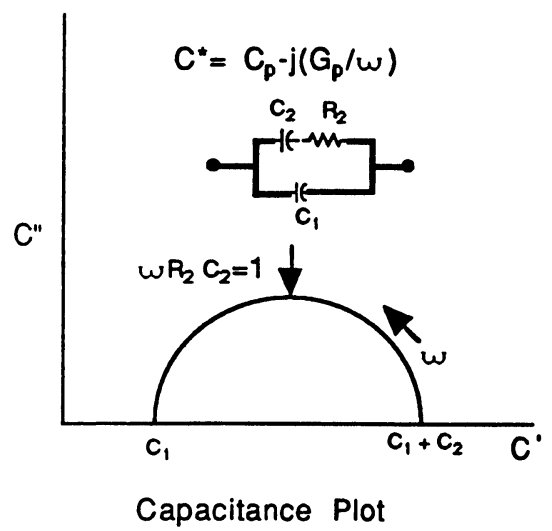

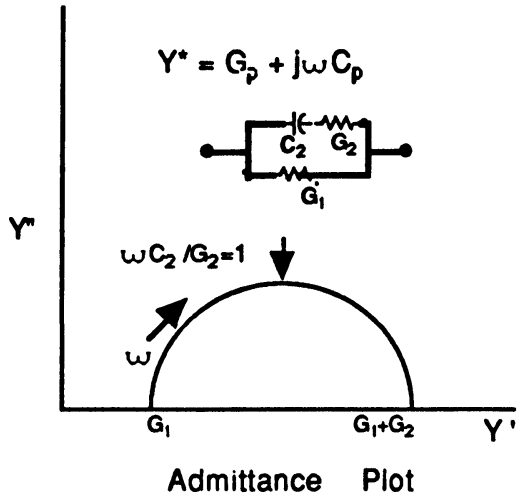

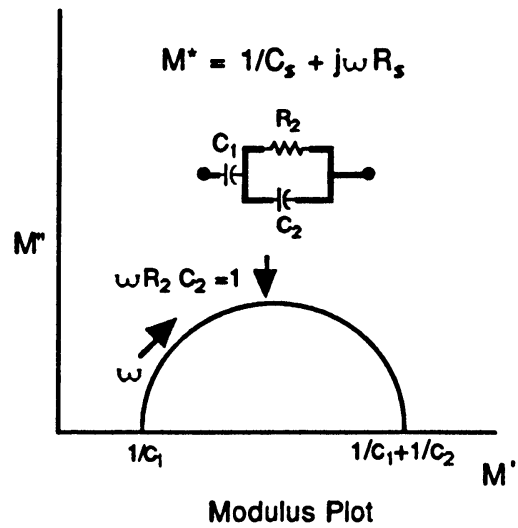

FIGURE 3 Sample plots with associated simplified equivalent circuit for each of the four complex planes. 
semicircle whose center lies on the $x$-axis is defined as a Debye (ideal) or Debyelike (nearly ideal) relaxation. In the event when the center lies below the $x$-axis, such a response is termed as non-ideal or non-Debye relaxation. ${ }^{2}$ The data-handling criteria involving proper fitting of the semicircular relaxation response and subsequent interpretation are important. The degree of accuracy in fitting the actual data points on the semicircular loci requires the nonlinear least squares analyses of these data. ${ }^{10-12}$ This approach ascertains proper numerical values of the equivalent circuit elements.

The complexity arises if a single relaxation is observed in more than one complex plane for the same set of data. Such cases can be delineated with the processing constituents, microstructural issues including phase distribution, role of dopants/ additives, nature of the electrical barriers, range of measurement values for dual or overlapped responses. Eventually, the dual-plane response restricts to one valid interpretation. The investigator has to choose the interpretation and determine the root of dual representation of the data. Further, the dual-plane response may influence the electrical parameters set for the application.

A higher degree of complexity is encountered in systems having multiple relaxations/semicircles. ${ }^{1,6-8,13}$ In such cases, it is found that the series circuits are best represented by $\mathrm{Z}^{*}$ - and $\mathrm{M}^{*}$-plots, while parallel circuits are best represented by $\mathrm{Y}^{*}$ - and $\mathrm{C}^{*}$-plots. The relative magnitudes of various circuit elements govern the choice between the $\mathrm{Z}^{*}$ and $\mathrm{M}^{*}$, or between the $\mathrm{Y}^{*}$ and $\mathrm{C}^{*}$. The complexity of systems that can be analyzed using LP/CPA is exemplified elsewhere. ${ }^{1}$ The case studies and limiting factors are also listed there.

For the LCR samples, only one relaxation is expected in the $\mathrm{Z}^{*}$-plane. The same relaxation can be found via Bode plots [for example: $\left|\mathrm{Z}^{*}\right|\left(\log \left|\mathrm{Z}^{*}\right|\right)$ or loss tangent $\left(\tan \delta=\mathrm{G}_{\mathrm{p}} / \omega \mathrm{C}_{\mathrm{p}}=\omega \mathrm{R}_{\mathrm{s}} \mathrm{C}_{\mathrm{s}}\right.$ ) versus frequency $\left.(\log f)\right]$. The sharpness of the relaxation obtained in the Bode plots is generally poor, and extrapolation due to the curve fitting causes this problem. A better degree of confidence is achieved via a complex plane semicircular fitting. This fitting ${ }^{13}$ provides:

1. voltage or current dividing equivalent circuit;

2. equivalent circuit elements (resistor, capacitor, inductor, etc.);

3. relaxation time (time-constant or peak-frequency);

4. left and right intercepts on the real axis;

5. chord or diameter (chord represents a depressed semicircle); and

6. depression angle $(\theta=h \pi / 2)$ or depression angle parameter $(0 \leq h \leq 1$ for Debye/non-Debye cases).

Each of these parameters lead to a meaningful interpretation related to the DUT. These parameters can be varied as a function of processing variables. Utilizing such information, the LP/CPA technique is applicable to a wide variety of heterogeneous composite material systems. Complex plane plotting, when combined with other information concerning the system under study, allows an equivalent circuit representation of the material. The various elements within the equivalent circuit can be used to follow the effects of important variables on specific regions within the microstructure. Thus, the microstructure-property-processing relationship can be established. 
The classical Bode plot involves magnitude (absolute modulus) of any one of the four complex electrical parameters versus measurement frequency. ${ }^{9}$ Often, each of the components (real or imaginary) and relevant phase angle may be plotted as a function of frequency. This is exercised to further understand the behavior of the in-phase and out-of-phase components. Depending on the nature of the curve on each Bode plane, an equivalent circuit analog may be developed. This approach reveals identical results to the LP/CPA for the simplified cases. Often BPA is not capable of resolving complex mechanisms and multiple competing phenomena. ${ }^{6-8}$ In this case, extraction of an equivalent circuit will not be meaningful as it may not match with the information revealed via the LP/CPA technique.

The spectroscopic analysis of the measured parameters, obtained in the immittance or phasor form under the non-equilibrium experimental conditions (such as: applied electrical field stress, temperature, compositional change, etc.), is also used by several investigators. ${ }^{14,15}$ A comparative study between the spectroscopic approach and LP/CPA technique reveals identical results. ${ }^{7-8}$ Figure 4 demonstrates

Measured form : $Y^{*}=G_{p}+j \omega C_{p}$

Admittance Spectroscopy

$\omega_{x}\left(=1 / \tau_{x}\right)$ is constant

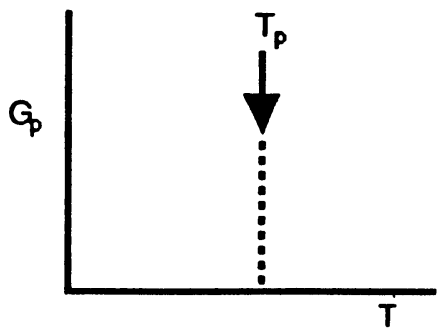

$T_{p}=$ Temperature where $G_{p}$ is maximum at a frequency $\omega_{x}$

$T_{x}=T=$ Measured Temperature

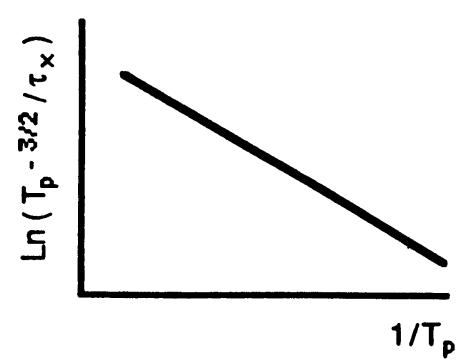

Lumped Parameter/Complex Plane Analysis $T_{x}$ is constant

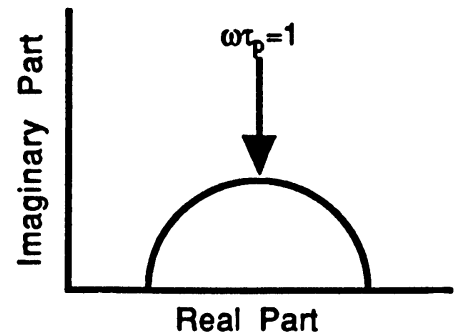

Complex Plane Plot

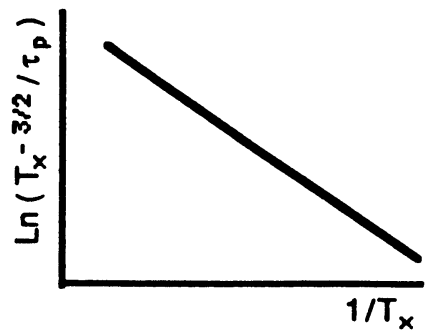

FIGURE 4 Demonstration of identical results obtained via spectroscopic approach and lumped parameter/complex. plane analysis. 
the verification of these two analytical methods. It is useful in the event a DUT does not yield a meaningful solution from the LP/CPA and BPA via the distortion in the response and departure from ideal features of the curves. This analytical method employs evaluation of the variation in the as-measured in-phase and outof-phase parameters (series or parallel) under non-equilibrium conditions. Such data are not displayed as the demonstration of the LP/CPA and BPA techniques are very effective for the specific samples examined here.

\section{EXPERIMENTAL APPROACH}

A real-world circuit element [i.e., resistor $(\mathrm{R})$ or capacitor/inductor $(\mathrm{C} / \mathrm{L})$ ] has parasitics that detract from the investigation of host-element's ideal characteristics. These parasitics, chiefly resulting from the non-ideal behavior (i.e., defects, imperfections, flaws, etc., in the material systems), increase the difficulty of performing accurate $\mathrm{R}$ or $\mathrm{C} / \mathrm{L}$ measurements. This situation allows the presence of unwanted minor contributing element(s) in the host-material. Certainly, the material systems and their fabricating technologies produce varying amounts of parasitics affecting host-element's (i.e., $\mathrm{R}$ or $\mathrm{C} / \mathrm{L}$ ) applications. The complex nature of a material system, comprised of the resistive and reactive elements, produces a phase shift between an applied voltage and the resulting current. In such a case, the measurement technique must account for this phase shift via insertion of an element either in series or parallel with the host-element as a reference to balance the unknown arm in the bridge system of the measuring instrument. ${ }^{16}$

The real-world elements possess real and reactive contributions resulting in an effective host-element for applications. As an example, real resistance can be measured at dc condition, but the result of such a measurement is the real value only under those specifically defined test-parameter and environmental conditions. A real resistance can be expressed as either a series or parallel equivalent value for applications. The relative magnitude depends on the degree of reactance associated with the host material system. For a highly conductive (i.e., extremely low real R) system, a 4-terminal/probe technique with constant-current source is necessary. ${ }^{16.17}$ This situation is averted here using a 2-terminal/probe technique with constantvoltage source for large resistance value. Environmental radiated interference causes measurement instabilities that can be remedied by proper shielding and/or grounding.

Considering the foregoing issues, while investigating smart (novel) materials for either a resistor or capacitor application, it is important to note that for a pure resistor (or a capacitor), a finite value of a capacitor (or a resistor) in parallel (or series) is necessary during the measurement processes. This arrangement constitutes terminal admittance, and complements balancing bridge arm of the measuring instrument. It must prevent instrumental damage from the electrical shock (i.e., short-circuit). In the event of the series addition of a buffer element to the DUT, it would provide terminal impedance. The electrical shock to the measuring instrument involving the bridge assembly may originate from the absence of either the in-phase or out-of-phase component between the terminals at the location of 
the sample holder. In general, smart materials inherit both resistance and capacitance due to their constituents, microstructures, processing methods, etc. Thus, these materials are the perfect systems for the ac small-signal measurements.

Two types of commercial resistors, designated as $\mathbf{T}_{1}$ and $\mathbf{T}_{2}$ and possessing various magnitudes $(100 \mathrm{k} \Omega-20 \mathrm{M} \Omega)$ are employed to demonstrate the usefulness of the IS via LP/CPA and BPA techniques. It is presumed that these resistors must possess low capacitance due to the type of their usage. These LCRs are believed to be different in composition in addition to the processing or method of fabrication. These are chosen because two distinguishable sets of electrical parameters arise from their resulting microstructure, although they may be designed for identical applications. The variation within the set of these parameters is likely to distinguish an overall performance.

The acquisition of the ac small-signal electrical data provides a quasi-equilibrium experimental condition and is assumed to be an excellent non-destructive operation for the entire measurement processes. These data are monitored as a function of frequency using an HP4192A LF Impedance Analyzer controlled by an HP86 computer/controller in the frequency range $5 \mathrm{~Hz} \leq f \leq 13 \mathrm{MHz}$. The preferred mode of measurement involves admittance as a function of frequency. To avert the effect of low frequency polarization during the measurement processes, the acquisition of the ac electrical data at these frequencies may be treated differently than at high frequencies. This special treatment may involve averaging admittance of certain reasonable number of observations for each frequency.

\section{EVALUATION OF THE RESISTORS}

The conduction mechanism and its influence on the electrical properties of perfect resistors (LCRs) are important to understand for application purposes. The exact constituents and processing history of a resistor dictate its properties. The LP/CPA technique allows us a clear understanding of the lumped response on the resulting composite/hybrid materials' behavior.

The measurement of each of these resistors involved an analysis of the ac smallsignal electrical data in the $\mathrm{Z}^{*}$-plane. A single semicircular relaxation is evident for these resistors. The form of the generic circuit for such relaxations, depicted in Figure 3, is an equivalent representation of the underlying mechanisms containing a R-C (resistor-capacitor) parallel combination. The left-intercept of the semicircle represents a series resistive element $\left(R_{1}\right)$ to this parallel combination.

Some interpretations are speculated from the nature of the electrical response in these resistors. Incorporating electrical behavior into the processing and microstructural information would certainly make the effort more meaningful.

\section{(a) $T_{1}$ Resistors}

The single relaxation response of the ac small-signal electrical data is displayed in Figure 5 for a $\mathbf{T}_{1}$ resistor. The minuscle noise in the semicircular loci at low frequencies is deleted for a clean appearance. This relaxation is attributed to the barrier layer polarization resulting from the barriers formed between the successive 


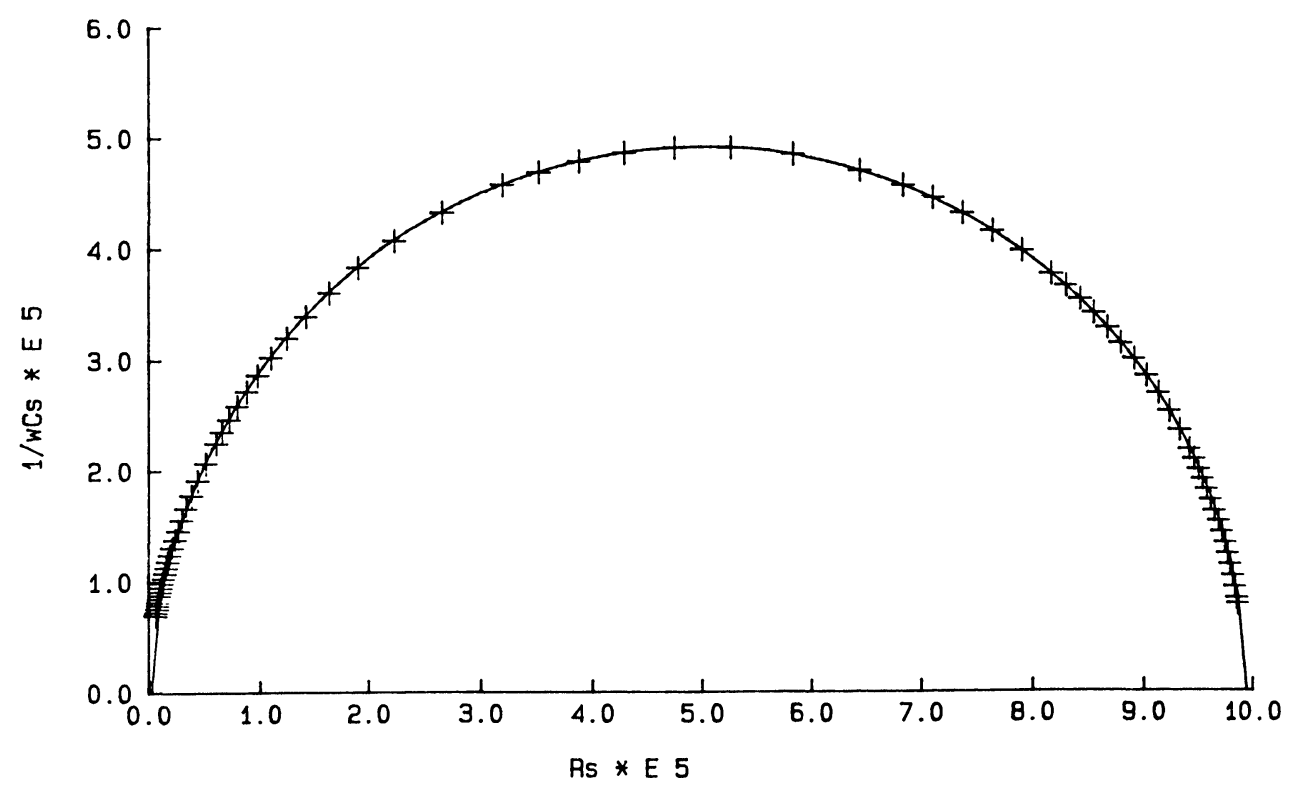

FIGURE 5 Semicircular fitting in the impedance plane $(37.5 \mathrm{kHz} \leq f \leq 6.75 \mathrm{MHz})$ for a $\mathbf{T}_{1}$ resistor (1 $\mathrm{M} \Omega, 1 \mathrm{Watt})$ at room temperature.

possible cation-related conductive particles. An insulating interfacial layer is felt to be most likely the source of this barrier. The barrier layer resistance $(R)$ is determined directly from the chord (in this case diameter) of the semicircular relaxation. This resistance correlates well with the value of the resistance designated by the manufacturers. The observed difference is less than $0.5 \%$. The corresponding barrier related capacitance $(\mathrm{C})$ is determined from the circuit time constant or relaxation time $(\tau=\mathrm{RC})$ obtained via the angular frequency associated with the peak $(\omega \tau=1)$ of the semicircular relaxation. The left-intercept $\left(R_{1}\right)$ of the semicircular relaxation most likely results from the lumped resistance of the cationrelated particles. The value of the left-intercept is found to be 3 or 4 orders in magnitude smaller than $R$ (i.e., $R_{1} \ll R$ ). The summation of these two resistances is a good approximation of the actual dc resistance of the specimen designated by the manufacturer. For all practical purposes, the measured dc resistance exhibits:

$\mathrm{R}_{\text {specimen }}=\mathrm{R}_{\mathrm{dc}}=\mathrm{R}+\mathrm{R}_{1} \approx \mathrm{R}$.

The presence of a minute depression angle in the semicircular relaxation indicates that this is nearly an ideal (Debye-like) system. This minute depression angle may have resulted from a possible error associated with the semicircular curve fitting. It is found to be much less than $1^{\circ}$, and does not seem to be a function of temperature or voltage. Furthermore, the Debye-like predominant response suggests that the series-parallel network of effective electrical paths between the terminals through the microstructure is homogeneous. Such behavior is likely to exist in an ideal trapfree single junction devices. ${ }^{6-8}$ 
The same impedance data when displayed in the Bode plane, depicted in Figure 6 , gives a straight line at high frequencies $\left(f \geq 10^{5} \mathrm{~Hz}\right)$. It represents the effect of the reactance of the total impedance yielding a slope close to $-0.999(-1$ is the ideal Debye value for the zero depression angle). At low frequencies, the flat line corresponds to the designated dc value of the resistance $\left(R_{\text {specimen }}\right)$ on the ordinate. The intersection of these two lines exhibiting as a transition is nearly rounded, and corresponds to the circuit time constant $(\tau)$ represented by the peak of the semicircle in the $Z^{*}$-plane. This rounded response often lead to a marginal error when compared to the semicircular fitting. The resistive element $R_{1}$ is not distinguishable via the Bode plot, presumably high frequency measurement in the range $f \sim 10^{7} \mathrm{~Hz}$ does not resolve it. The exact value of $R_{1}$ is delicate and not precisely obtainable from the Bode plot within this range of measurement frequency.

When each resistor is subjected to the ac small-signal stress as a function of temperature $\left(300 \leq \mathrm{T} \leq 375^{\circ} \mathrm{K}\right)$, it is found that $\mathrm{R}_{1}$ increases with increasing temperature. Using the model of Halder and Snyder, ${ }^{18}$ a positive TCR is found to be ranging between $24 \times 10^{-3} /{ }^{\circ} \mathrm{K}$ and $49 \times 10^{-3} /{ }^{\circ} \mathrm{K}$ for high- and low-magnitude resistors, respectively. The origin of the difference between the TCRs is not exactly known. However, the method of extraction of the left intercept (Figure 2) from the curve fitting procedure is unlikely to be the source of this observation.

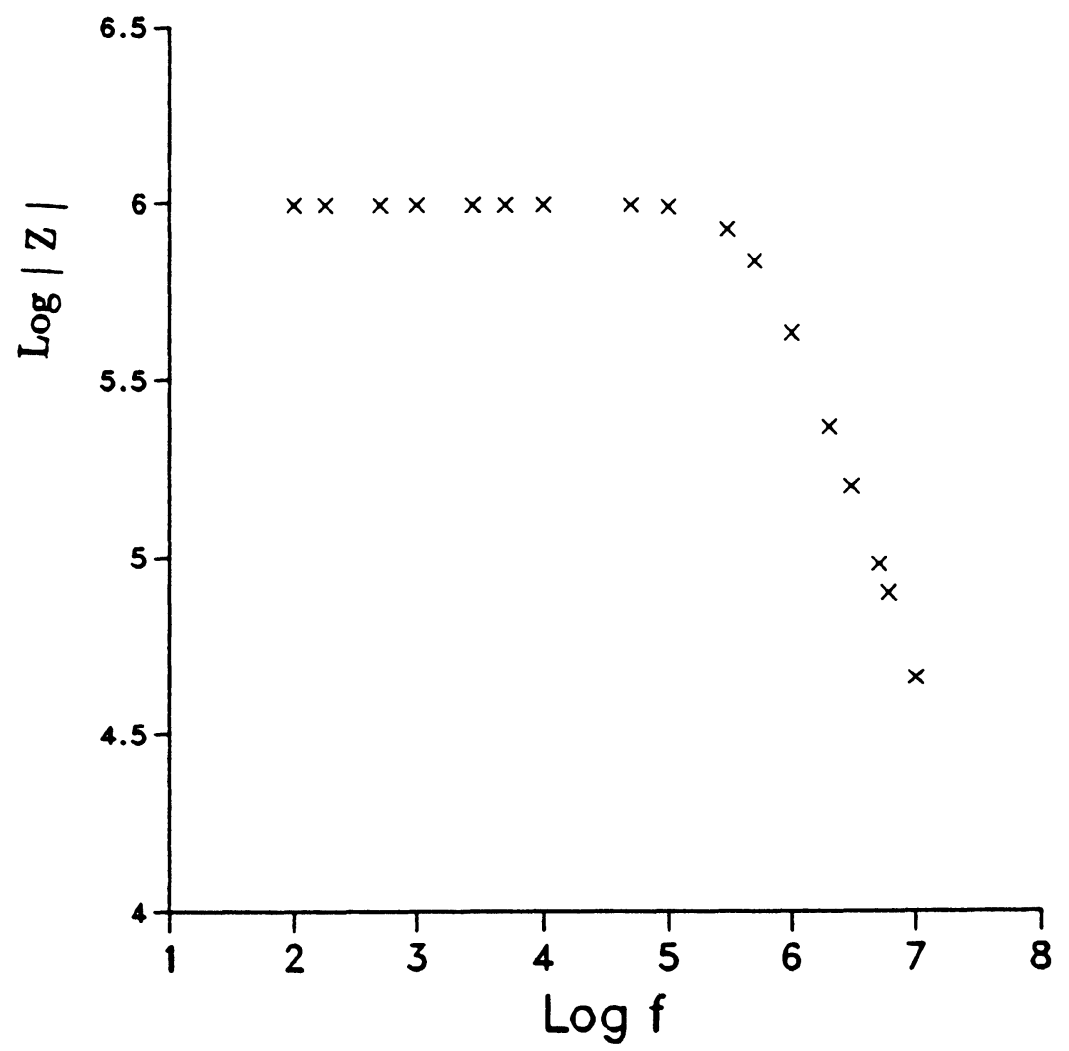

FIGURE 6 Bode plot for $\mathbf{T}_{1}$ resistor $\left(\left|Z^{*}\right|=|Z|\right)$ for the same data displayed in Figure 4. 
The barrier layer resistance, R, obtained from the Bode plot or the chord of the semicircle, and the time constant $(\tau)$ associated with it, for all cases, are found to be nearly independent of temperature (Figure 7). These imply that the corresponding capacitance $(\mathrm{C})$ is also invariant with temperature. These equivalent circuit elements meet manufacturer's designation for each resistor. In addition, the dc current-voltage measurement as a function of temperature indicates no variation in the value of the resistance. Thus, the TCR of $\mathrm{R}$ (extracted from the dc measurement and LP/CPA) is found to be either too small to detect or near zero. The temperature dependence of the resistance, $R_{1}$, does not influence the dc measurement within the experimental situation, as it is masked due to the large value of $R$. The resistance $R_{1}$ is so small that is has no practical influence on the application of the $\mathbf{T}_{1}$ resistor in the aforementioned temperature range. However, $\mathbf{R}_{1}$ follows an Arrhenius relationship yielding an activation energy $\approx 0.2 \mathrm{eV}$ or less (Figure 8).

(b) $T_{2}$ Resistors

The dispersion of the immittance with ac frequency for the $\mathbf{T}_{2}$ resistors is nearly identical to the $\mathbf{T}_{1}$ resistor. A single relaxation response of the ac small-signal

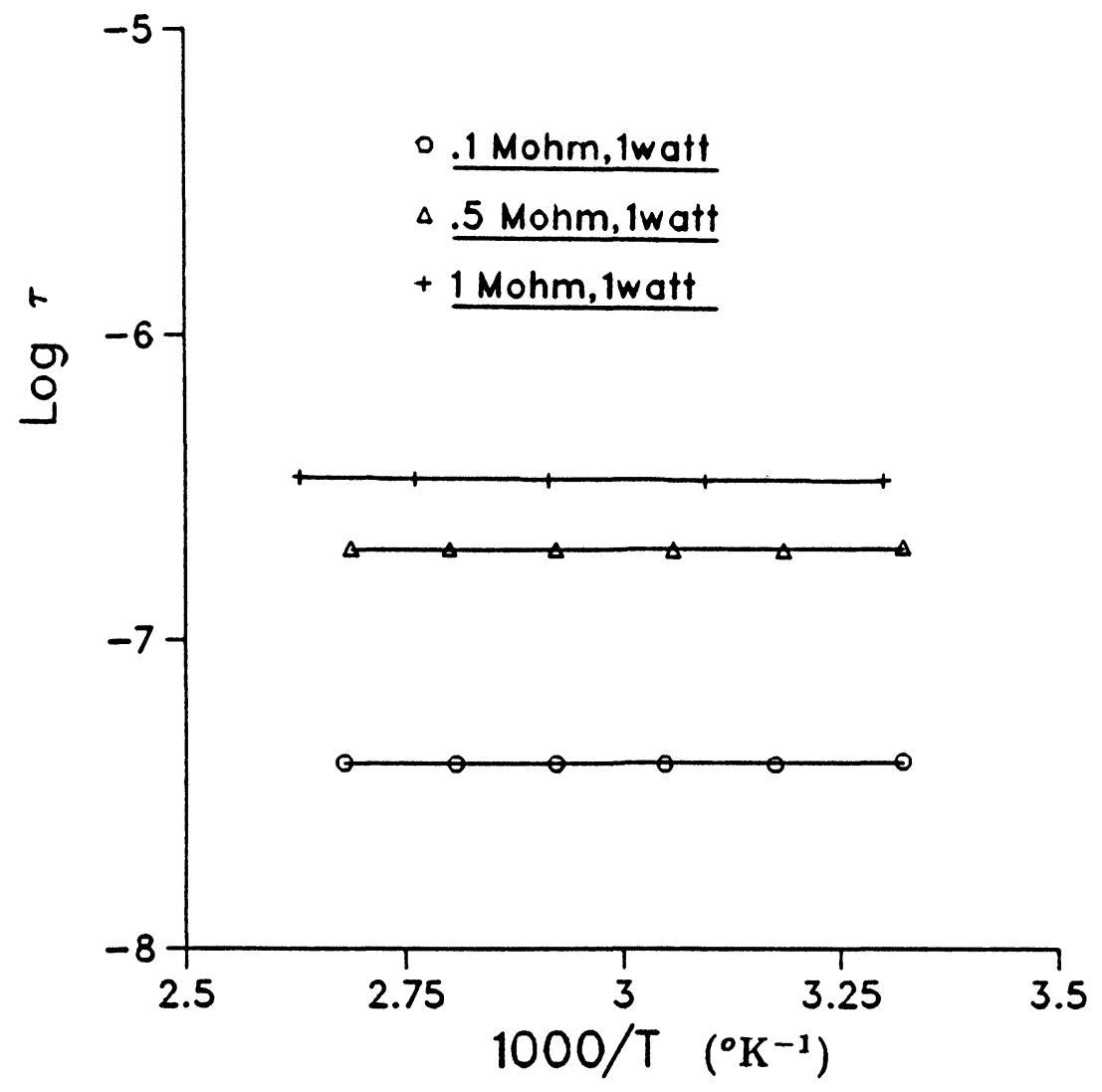

FIGURE 7 Temperature dependence of $\tau$ for $\mathbf{T}_{1}$ resistors. 


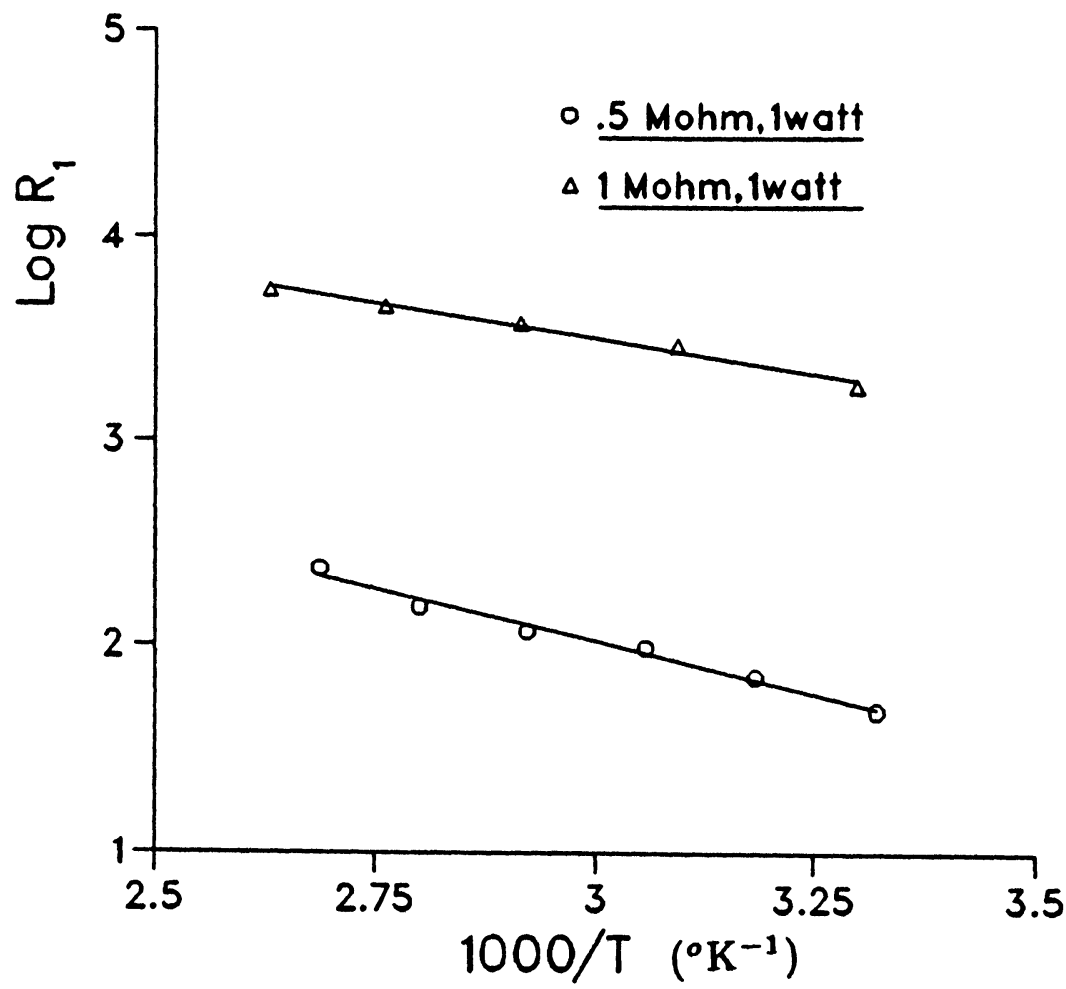

FIGURE 8 Temperature dependence of $R_{1}$ for $\mathbf{T}_{1}$ resistors.

electrical data is evident in the impedance plane for these resistors. This is attributed to the barrier layer polarization. The rationale for the components making up the equivalent circuit for the relaxation is similar to that used for $\mathbf{T}_{1}$ resistors. The barrier layer is thought to exist between successive particles and to consist of thin layers. The barrier resistance $(\mathrm{R})$ is determined from the chord of the semicircular relaxation, and the corresponding capacitance $(\mathrm{C})$ is extracted from the circuit relaxation time $(\tau)$. The observed resistance is within $0.5 \%$ of the manufacturer's value.

In these resistors, within the error limit, the left intercept $\left(R_{1}\right)$ is near zero. This component appears to be too small to be determined in the $\mathrm{Z}^{*}$-plane as a function of temperature and voltage. However, the equivalent circuit representation for this resistor is similar to that shown in Figure 3 without the resistor $R_{1}$. Essentially, the effective resistance, designated by the manufacturer, is exclusively contributed by the element $\mathrm{R}$. The capacitance, $\mathrm{C}$, obtained from the equivalent circuit, ranges between 0.28 and $0.36 p \mathrm{~F}$. It descreses with increase in manufacturer designated value of the resistance. This may be due to the fact that at lower magnitudes of resistance, the loading of particles is much more than the loading in the higher magnitude resistors. This indicates lower number of inter-particle junctions for the higher magnitude resistors resulting in a decrease in the capacitance. 
The presence of a finite depression angle in the semicircular relaxation indicates that this is not an ideal system. This finite (non-zero) depression angle (11 $\leq \theta \leq$ $14^{\circ}$ or $0.122 \leq h \leq 0.155$ ) indicates a distribution of relaxation time originating from an inhomogeneity in the microstructural system, and often referred to as nonDebye response..$^{2,8,19}$ In addition, the inhomogeneity is likely to be associated with the capacitive component represented via the depressed ordinate parameter in the $\mathrm{Z}^{*}$-plane representation. A small variation in the depression angle for these resistors is not too definitive, but indicative of a fluctuation in the degree of inhomogeneity within the effective conducting path(s) between the terminals. The minuscule increase in the depression angle (wihtin $1^{\circ}$ ) in the tolerating temperature range $300 \leq \mathrm{T} \leq 375^{\circ} \mathrm{K}$ suggests that the distribution of activation energies and/ or relaxation times involving the conduction path(s) is slightly approaching the degree of nonuniformity. The summary of the ac parameters, obtained within the degree of accuracy and confidence level, is provided in Table 1.

The relaxation resistance component $(\mathrm{R})$ may be portrayed as the total resistance specified by the manufacturer. It increases with increasing temperature in the range $300 \leq \mathrm{T} \leq 375^{\circ} \mathrm{K}$, and positive TCR values are observed between $932 \times 10^{-6} /{ }^{\circ} \mathrm{K}$ (for the high resistance value) and $1414 \times 10^{-6} /{ }^{\circ} \mathrm{K}$ (for the low resistance value). The dc measurement yields similar positive TCR values for each resistor. This is probably resulting from the expansion of the thin layers, which decreases contact pressure between the particles, and thereby increases the value of the resistance. ${ }^{20}$

The temperature dependence of $\tau$ yields a thermal activation energy $\left(\mathrm{E}_{\tau}\right)$ ranging between 0.01 and $0.02 \mathrm{eV}$. The related components ( $\mathrm{R}$ and $\mathrm{C}$ ) are also thermally activated. Nearly $60-80 \%$ of this activation energy is associated with the capacitive element. Taking this response into consideration in conjunction with the presence of the depression angle, it implies that the nature of this semicircular relaxation is more complex. A similar situation is recently reported for the intrinsic trapping response in the zinc oxide varistors. ${ }^{8}$

It may be noted here that the variation in the measured parallel capacitance, $\mathrm{C}_{\mathrm{p}}$, as a function of frequency, does not seem to change with increasing temperature at frequencies $f \geq 10^{3} \mathrm{~Hz}$. Also, the small variation in $\mathrm{C}_{\mathrm{p}}$, observed at the low frequencies $\left(f \leq 10^{3} \mathrm{~Hz}\right)$ in the aforementioned temperature range, is attributed to a possible change occurring within the operative electrical paths and/or effective conducting area among the inter-particle barriers at elevated temperatures. It is a typical characteristic of the non-Debye systems. ${ }^{8}$ From the application standpoint, this type of resistor is certainly sensitive at the ambient temperature. However, this resistor can have high frequency applications at elevated temperatures for a specific value to be achieved.

TABLE 1

Equivalent circuit elements with their corresponding depression angle for the $\mathbf{T}_{2}$ resistors at room temperature $\left(\approx 300^{\circ} \mathrm{K}\right)$.

\begin{tabular}{lcccccr}
\hline $\mathrm{R}(\mathrm{M} \Omega)$ & 0.1 & 0.5 & 1.0 & 5.2 & 10.0 & 20.0 \\
\hline $\mathrm{C}(p \mathrm{~F})$ & 0.367 & 0.362 & 0.324 & 0.295 & 0.280 & 0.276 \\
\hline $\boldsymbol{\theta}\left({ }^{\circ}\right)$ & 11.133 & 11.998 & 12.403 & 13.711 & 13.937 & 14.333 \\
\hline
\end{tabular}




\section{CONCLUSIONS}

Various aspects of the immittance spectroscopy in the applications of the engineering material systems are discussed. The Debye-like and non-Debye samples for resistor applications are demonstrated utilizing a complete set of analytical technique provided by this spectroscopic approach. The semicircular relaxation obtained in the $\mathrm{Z}^{*}$-plane represents barrier layer polarization in both type of resistors. The barrier resistance, obtained via the semicircular relaxation, is essentially the designated value specified by the manufacturers. For the $\mathbf{T}_{1}$ resistors, the left intercept of this relaxation is attributed to the tentative lumped resistance of the possible cation-related particles and found to be thermally activated. The operative conduction paths in these resistors are homogeneous via the presence of a Debye-like near-zero depression angle in the semicircular relaxation. The $\mathbf{T}_{2}$ resistors exhibited an inhomogeneous nature of the conduction processes via the existence of a finite depression angle. The relaxation time and its constituent elements for the $\mathbf{T}_{1}$ resistors are not thermally activated. These parameters of the $\mathbf{T}_{2}$ resistors exhibited similar response. The postulated equivalent circuit elements represent competing phenomena and are operative within these resistors. The LP/CPA and BPA techniques provide meaningful solutions to several problems when various material systems are used in characterizing, designing, and making either resistors or capacitors for the component applications.

\section{REFERENCES}

1. M.A. Seitz, "Study of Heterogeneous Composite Materials via Lumped Parameter/Complex Plane Analysis," Int. J. Hybr. Microelectron., 3, 1 (1980); J.J. Ackman and M.A. Seitz, "Methods of Complex Impedance Measurements in Biologic Tissue," CRC Crit. Rev. Biomed. Engr., 11, 281 (1984).

2. M.A. Alim and M.A. Seitz, "Singular Nature of Preferential Conducting Paths in ZnO-Based Varistors," J. Am. Ceram. Soc., 71, C246 (1988).

3. C.J. Ting, C.J. Peng, H.Y. Lu and S.T. Wu, "Lanthanum-Magnesium and Lanthanum-Manganese Donor-Acceptor Codoped Semiconducting Barium Titanate," J. Am. Ceram. Soc., 73, 329 (1990).

4. E.A. Cooper, T.O. Mason, M.E. Biznek and U. Balachandran, "Impedance Spectra During Solid State Powder Reactions of Oxide Superconductors," J. Am.Ceram. Soc., 73, 154 (1990).

5. J.E. Bauerle, "Studies of Solid Electrolyte Polarization by a Complex Admittance Method," J. Phys. Chem. Sol., 30, 2657 (1969).

6. A.M. Azad, L.B. Younkman, S.A. Akbar and M.A. Alim, "Characterization of $\mathrm{TiO}_{2}$-Based Sensor Materials by Immittance Spectroscopy," J. Am. Ceram. Soc., 77, 481 (1994).

7. M.A. Alim, M.A. Seitz and R.W. Hirthe, "Complex Plane Analysis of Trapping Phenomena in Zinc Oxide Based Varistor Grain Boundaries," J. Appl. Phys., 63, 2337 (1988).

8. M.A. Alim, "Admittance-Frequency Response in Zinc Oxide Varistor Ceramics," J. Am. Ceram. Soc., 72, 28 (1989).

9. H.W. Bode, Network Analysis and Feedback Amplifier Design (Van Nostrand, New York, 1945).

10. D.W. Marquardt, "An Algorithm for Least-Squares Estimation of Nonlinear Parameters," J. Soc. Indust. Appl. Math. (Am. Math.), 11, 431 (1963).

11. Y.T. Tsai and W.H. Whitmore, "Nonlinear Least-Square Analyses of Complex Impedance and Admittance Data for Solid Electrolytes,” Sol. St. Ion., 7, 129 (1982).

12. W. Schreiner, M. Kramer, S. Krischer and Y. Langsam, "Nonlinear Least-Squares Fitting," PC Tech Journal (IBM), pp 170-185, May 1985.

13. M.A. Alim, "Comment on 'Temperature Dependence of Electrical Conductivity in Polycrystalline Tin Oxide," J. Am. Ceram. Soc., 74, 1757 (1991). 
14. F. Greuter and G. Blatter, "Electrical Properties of Grain Boundaries in Polycrystalline Compound Semiconductors," Semicond. Sci. Technol., 5, 111 (1990); F. Greuter, G. Blatter, M. Rossinelli and F. Stucki, "Conduction Mechanism in ZnO Varistors: An Overview," Ceramic Transactions: Advances in Varistor Technology, 3, 31 (1989); G. Blatter and F. Greuter, "Carrier Transport Through Grain Boundaries in Semiconductors," Phys. Rev. B, 33, 3952 (1986).

15. Y. Shim and J.F. Cordaro, "Admittance Spectroscopy of Polycrystalline $\mathrm{ZnO}-\mathrm{Bi}_{2} \mathrm{O}_{3}$ and $\mathrm{ZnO}$ BaO Systems," J. Am. Ceram. Soc., 71, 184 (1988); J.F. Cordaro, Y. Shim and J.E. May; "Bulk Electron Traps in Zinc Oxide Varistors," J. Appl. Phys., 60, 4186 (1986).

16. B. Botos, "Designer's Guide to R-C-L (Resistance-Capacitance-Inductance) Measurements: 1-4 Parts," EDN (Hewlett-Packard Reprint), 1-32, June 5 (1979).

17. M.A. Seitz, R.T. McSweeney and W.M. Hirthe, "A Technique for Determining the Dielectric Behavior of High Conductivity Materials," Rev. Sci. Instr., 40, 826 (1969).

18. N.C. Halder and P.J. Snyder, "Measurement of the Tunneling and Hoping Parameters in $\mathrm{RuO}_{2}$ Thick Film Resistors," Electrocomp. Sci. and Technol., 11, 123 (1984).

19. A. van Roggen, "Distribution of Relaxation Times and Their Argand Diagrams," IEEE Trans. Elect. Insul., EI-5, 47 (1970).

20. C.L. Wellerd, Resistance and Resistors (McGraw-Hill Book Company, Inc., New York, 1960). 

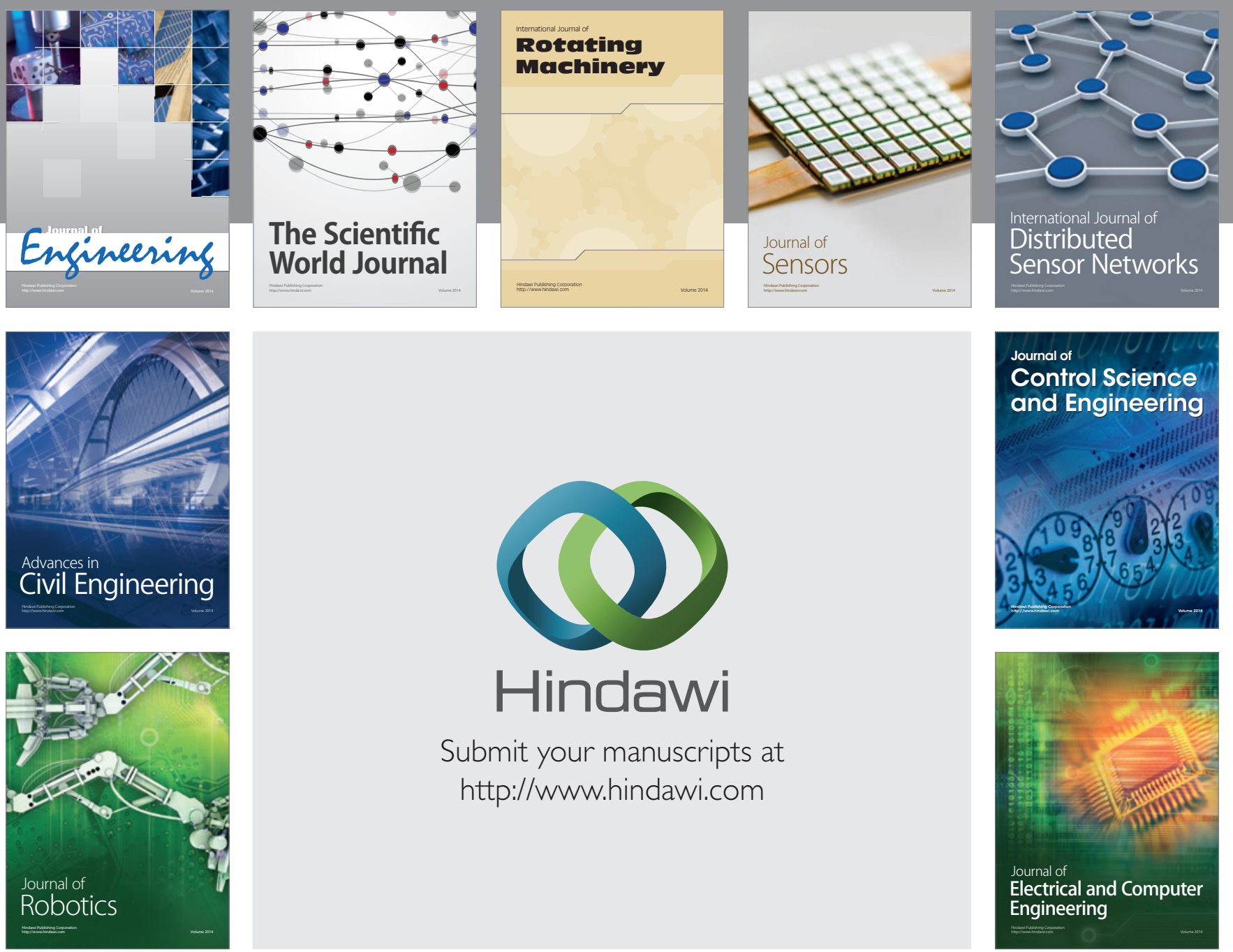

Submit your manuscripts at

http://www.hindawi.com
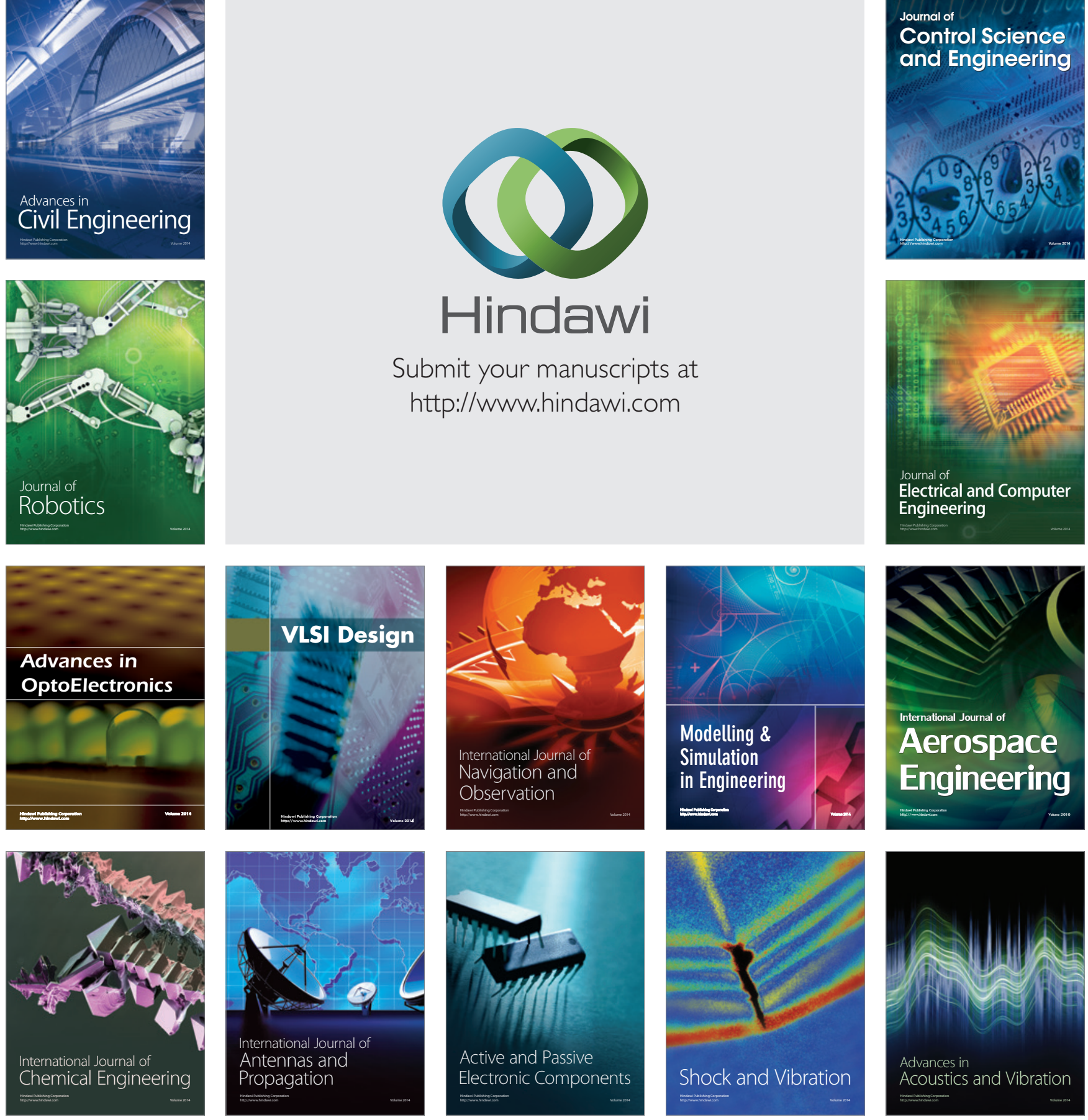